\title{
Long-term experiences (max. 22 years) with 38 antisiphon devices
} A Aschoff*1, D Biedermann ${ }^{1}$, J Ludwig ${ }^{1}$, A El Tayeh ${ }^{1}$, N Biedermann ${ }^{1}$, A Piotrowicz ${ }^{1}$ and $P$ Kremer ${ }^{2}$

\author{
Address: ${ }^{1}$ University of Heidelberg, Dept of Neurosurgery, Im Neuenheimer Feld 400, 60120 Heidelberg, Germany and ${ }^{2}$ Dept. of Neurosurgery, \\ Hospital Hamburg-Haidberg, Germany \\ Email: A Aschoff* - Alfred_Aschoff@med.uni-heidelberg.de \\ * Corresponding author
}

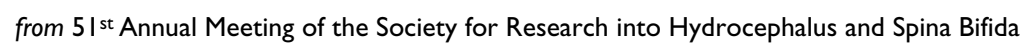
Heidelberg, Germany. 27-30 June 2007

Published: 20 December 2007

Cerebrospinal Fluid Research 2007, 4(SuppI I):S52 doi:I0.II86/I743-8454-4-SI-S52

This abstract is available from: http://www.cerebrospinalfluidresearch.com/content/4/SI/S52

(c) 2007 Aschoff et al; licensee BioMed Central Ltd.

\section{Background}

In 1973 Portnoy and Schulte developed the antisiphondevice for prevention of over drainage, a supplementary valve with a variable resistance depending on body verticalization respect. The variable suction of the water column in distal catheter. Clones were the SCD (Medtronic) and SLD (Radionics). All were assembled to other DPvalves or integrated in bodies combined with conventional valves (Delta, Novus). Unfortunately many cases with enigmatous hydraulic problems, especially underdrainage, but also over drainage were observed. In 1989 we identified the cause, the excessive susceptibility on external pressure, e.g. scars. For the compensation of these disturbing influences some users combined the antisiphons with adjustable Sophy- or Medos-valves. Unfortunately evaluations of these combinations, which anticipated the later Strata-valve, were not published. Generally long-term results of antisiphons are rarities.

\section{Materials and methods}

We evaluated retrospectively 38 patients with antisiphons 4-22 years after implantation, of them 18 with adjustable valves. 5 had telemetric ICP sensors.

\section{Results}

$12 / 38$ antisiphons are still implanted (survival 32\%), each 3 with good, fair, insufficient and poor condition. 20 patients were converted to gravitational, four to adjustable and one to a simple DP valve. Two became shunt free.
The quote of sufficient results in long-term counted 7.9\% $(3 / 38)$.

\section{Discussion}

34 years after introduction of antisiphons exist only two prospective randomized series with cumulative 430 Delta/Strata-patients (Drake 98, Kestle 05); both showed no differences compared to conventional valves in short term. Generally we found surprisingly few studies, approx. 50\% with standard and 50\% with inferior results (Miyake 99), but only mini-samples with superior data in short follow-up (Kondageski 07). 8 independent laboratory studies confirmed the excessive susceptibility on external pressure, which plausibly explains the clinical problems.

\section{Conclusion}

In short term, antisiphons show mostly no difference to other valves, sometimes they are inferior. In the course of time the specific problems increase and lead to a poor survival- and success-quote. Adjustable valves may damp, but are unable to compensate this effect. 